\title{
Decompositions of suspensions of spaces involving polyhedral products
}

\author{
KOUYEMON IRIYE \\ DAISUKE KISHIMOTO
}

\begin{abstract}
Two homotopy decompositions of suspensions of spaces involving polyhedral products are given. The first decomposition is motivated by the decomposition of suspensions of polyhedral products by Bahri, Bendersky, Cohen and Gitler, and is a generalization of a retractile argument of James. The second decomposition is on the union of an arrangement of subspaces called diagonal subspaces, and generalizes a result of Labassi.
\end{abstract}

$55 \mathrm{P} 15 ; 55 \mathrm{U} 10,52 \mathrm{C} 35$

\section{Introduction}

A polyhedral product is a space constructed from a collection of pairs of spaces in accordance with the combinatorial information of a given abstract simplicial complex, where the collection is labeled by vertices of the given simplicial complex. By definition, polyhedral products are related to fundamental objects in combinatorics, geometry, and topology such as Stanley-Reisner rings and their derived algebras, graph products of groups (eg right-angled Artin and Coxeter groups), quasitoric manifolds, coordinate subspace arrangements, higher-order Whitehead products, and so on. The aim of this paper is to provide two kinds of homotopy decompositions of suspensions of spaces involving polyhedral products: one is a generalization of the decompositions of Bahri, Bendersky, Cohen and Gitler [5] and Adem, Bahri, Bendersky, Cohen and Gitler [1], and the other is a decomposition of the union of arrangements of special subspaces called diagonal subspaces which include polyhedral products as subspaces. We briefly explain the backgrounds of these decompositions.

An important property of polyhedral products is the existence of retractions onto certain "sub"polyhedral products, where this type of retraction property also appears in other contexts; see Adem and Cohen [2], Adem, Cohen and Gómez [3], Adem, Cohen and Torres Giese [4] and Kamiyama and Tsukuda [15]. By using this retraction property, Bahri, Bendersky, Cohen, and Gitler [5] gave a decomposition of suspensions of 
polyhedral products, and we aim to generalize this decomposition. It is actually obtained by the retractile argument due to James [13], which provides a decomposition of suspensions of spaces satisfying a certain retraction property, and we will generalize the retractile argument in our first decomposition. Our decomposition has a naturality which cannot be obtained by the retractile argument, and recovers, of course, a decomposition of suspensions of polyhedral products by Bahri, Bendersky, Cohen, and Gitler [5] and also the decomposition of suspensions of simplicial spaces by Adem, Bahri, Bendersky, Cohen, and Gitler [1]. We here note that in [1] it is pointed out that the decomposition of suspensions of polyhedral products can be obtained from the decomposition of suspensions of simplicial spaces, but polyhedral products do not seem to fit into the context of simplicial spaces.

The second space which we decompose is the union of an arrangement of special subspaces called diagonal subspaces, which includes important subspace arrangements such as braid arrangements, where we abbreviate this union as a diagonal arrangement. The decomposition of a suspension of diagonal arrangements was formerly studied by Labassi [18] in a special case, and Sadok Kallel posed a question whether the result of Labassi can be generalized to general diagonal arrangements under a certain dimensional condition imposed on the special case of Labassi. We give an affirmative answer to this question with our second decomposition. These diagonal arrangements include special polyhedral products as subspaces, though in general we cannot describe properties of these polyhedral products as subspaces of the diagonal arrangements, ie properties of the inclusion. But under a certain dimensional condition, we can describe properties of the inclusion which enable us to prove the decomposition.

Acknowledgements The authors are grateful to the referees for useful advises and helpful comments, where they pointed out that it is sufficient to assume retractability of $\Sigma X$ instead of $X$ in Theorem 2.3 and showed a generalization mentioned in Remark 2.4. The first author is supported by JSPS KAKENHI (number 26400094), and the second author is supported by JSPS KAKENHI (number 25400087).

\section{Retractile spaces over posets}

In this section we consider a space over a poset with natural retractions, and prove a decomposition of a suspension of its certain colimit. To explain what we are going to do, we start with a simple example. Consider the diagram

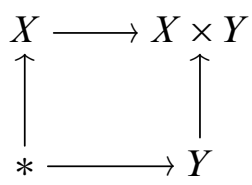


of spaces. Then we see that every arrow has a retraction, and it induces a filtration

$$
* \subset X \vee Y \subset X \times Y \text {. }
$$

By the above retractions, the filtration splits after a suspension to yield the decomposition

$$
\Sigma(X \times Y) \simeq \Sigma(X \vee Y) \vee \Sigma(X \wedge Y)
$$

which is natural with respect to $X$ and $Y$. The aim of this section is to generalize this situation. Let $P$ be a poset. We regard $P$ as a category by pointing upward, that is, for $p, q \in P, p \rightarrow q$ in the category means $p \leq q$ in the poset. We assume two conditions on $P$ :

(1) $P$ is graded, ie $P=\coprod_{n \geq 0} P^{n}$ as sets and for $p \in P^{n}$ and $q \in P^{m}, p<q$ implies
$n<m$. (2) $P$ is a lower semilattice, ie any $p, q \in P$ have the greatest lower bound $p \wedge q$.

Let $X$ be a space over $P$ which is a functor from $P$ to the category of pointed topological spaces. A map between spaces over $P$ is a natural transformation as usual. The grading of $P$ defines a filtration

$$
X^{\underline{0}} \subset X^{\underline{1}} \subset \cdots \subset X^{\underline{n}} \subset X^{\underline{n+1}} \subset \cdots,
$$

where $X^{\underline{n}}=\left.\operatorname{colim} X\right|_{P{ }_{n}}$ for the restriction $\left.X\right|_{P{ }_{n}}$ of $X$ to the subcategory $P^{\leq n}:=$ $\bigsqcup_{0 \leq k \leq n} P^{k}$. We say that $X$ is $n$-cofibrant if the canonical map $X^{\underline{i}} \rightarrow X \underline{i+1}$ is a cofibration for $i=0, \ldots, n-1$. There is a sufficient condition for the $n$-cofibrancy.

Lemma 2.1 (cf [19]) If all arrows of $\left.X\right|_{P \leq n}$ are cofibrations, $X$ is $n$-cofibrant.

We now define natural retractions in the diagram $X$, and state the main result of this section.

Definition 2.2 We say that $X$ is retractile if every arrow $\iota_{q, p}: X_{p} \rightarrow X_{q}$ admits a retraction $\rho_{p, q}$ satisfying

$$
\rho_{p, r} \circ \iota_{r, q}=\rho_{p, q} \quad \text { and } \quad \rho_{p, r}=\rho_{p, q} \circ \rho_{q, r} \quad \text { for } \quad p<q<r .
$$

Let $X, Y$ be retractile spaces over $P$. We say that a map $f: X \rightarrow Y$ of spaces over $P$ preserves retractions if $\rho_{p, q}^{Y} \circ f_{q}=f_{p} \circ \rho_{p, q}^{X}$ for any $p<q \in P$, where $\rho_{p, q}^{X}$ and $\rho_{p, q}^{Y}$ are the retractions of $X$ and $Y$, respectively. Put $X(p):=X_{p} /\left.\operatorname{colim} X\right|_{P_{<p}}$ for $p \in P$, where $P_{<p}:=\{q \in P \mid q<p\}$. 
Theorem 2.3 Let $X$ be a space over a graded lower semilattice $P$. If $X$ is $n$-cofibrant and $\Sigma X$ is retractile, then there is a homotopy equivalence

$$
\Sigma X^{\underline{n}} \simeq \Sigma \bigvee_{p \in P \leq n} X(p)
$$

which is natural with respect to maps of spaces over $P$ preserving retractions.

Remark 2.4 We can generalize Theorem 2.3 by weakening the condition to that there are maps $\bar{\rho}_{p, q}: \Sigma X_{q} \rightarrow \Sigma X(p)$ for any $q>p \in P$ such that the composite

$$
\Sigma X_{p} \stackrel{\Sigma \iota_{q, p}}{\longrightarrow} \Sigma X_{q} \stackrel{\bar{\rho}_{p, q}}{\longrightarrow} \Sigma X(p)
$$

is the quotient map and $\bar{\rho}_{p, q} \circ \Sigma \iota_{q, r}=\bar{\rho}_{p, r}$ for $p<r<q \in P$, where $\iota_{p, q}$ is an arrow in $X$. Indeed, we can construct a quotient map $\bar{\rho}_{p}^{m}: X^{\underline{m}} \rightarrow X(p)$ for $p \in P^{k}$ with $k \leq m$ satisfying a property analogous to Lemma 2.5 , so the proof of Theorem 2.3 works for this situation.

The rest of this section is devoted to the proof of this theorem, and we prepare two lemmas.

Lemma 2.5 For $p \in P^{k}$ with $k \leq m$, there is a retraction $\rho_{p}^{m}: X^{\underline{m}} \rightarrow X_{p}$ of the canonical map $X_{p} \rightarrow X^{\underline{m}}$ satisfying

$$
\rho_{p}^{m} \circ i=\rho_{p}^{\ell}
$$

for $k \leq \ell \leq m$ and the canonical map $i: X^{\ell} \rightarrow X^{\underline{m}}$.

Proof Let $\iota_{r, q}: X_{q} \rightarrow X_{r}$ be the arrow in $X$ for $q<r \in P$. Fix $p \in P^{k}$. Since $P$ is a lower semilattice, we can define a space $Y$ over $P$ by putting $Y_{q}=X_{p \wedge q}$ and the arrow $Y_{q} \rightarrow Y_{r}$ to be $\iota_{p \wedge r, p \wedge q}$. Then the map $\theta_{q}:=\iota_{q, p \wedge q}: Y_{q}=X_{p \wedge q} \rightarrow X_{q}$ defines a map $\theta: Y \rightarrow X$ of spaces over $P$. Indeed for $q<r$, we have

$$
\theta_{r} \circ \iota_{p \wedge r, p \wedge q}=\iota_{r, p \wedge r} \circ \iota_{p \wedge r, p \wedge q}=\iota_{r, p \wedge q}=\iota_{r, q} \circ \iota_{q, p \wedge q}=\iota_{r, q} \circ \theta_{q} .
$$

The map $\tau_{q}:=\rho_{p \wedge q, q}: X_{q} \rightarrow X_{p \wedge q}=Y_{q}$ also defines a map $\tau: X \rightarrow Y$ of spaces over $P$ since for $q<r$, we have

$$
\tau_{r} \circ \iota_{r, q}=\rho_{p \wedge r, r} \circ \iota_{r, q}=\iota_{p \wedge r, p \wedge q} \circ \rho_{p \wedge q, q}=\iota_{p \wedge r, p \wedge q} \circ \tau_{q} .
$$

By definition, we have $\tau \circ \theta=1_{Y}$ and $Y^{\underline{n}}=X_{p}$ for $n \geq k$. Thus the induced map $X^{\underline{m}} \rightarrow Y^{\underline{m}}=X_{p}$ from $\tau$ is the desired retraction. 
Lemma 2.6 [10, Theorem 4.2] If there is a homotopy retraction $r$ of the suspension $\Sigma f$ of a cofibration $f: A \rightarrow B$, then the map

$$
r+\Sigma \pi: \Sigma B \rightarrow \Sigma A \vee \Sigma(B / A)
$$

is a homotopy equivalence, where $\pi: B \rightarrow B / A$ is the projection.

Proof of Theorem 2.3 We show that the map

$$
\sum_{p \in P \leq n} \Sigma \pi_{p} \circ \rho_{p}^{n}: \Sigma X^{\underline{n}} \rightarrow \Sigma \bigvee_{p \in P \leq n} X(p)
$$

is a homotopy equivalence, which implies the desired naturality, where $\rho_{p}^{n}$ is as in Lemma 2.5 for $\Sigma X$ and $\pi_{p}: X_{p} \rightarrow X(p)$ is the projection. Let $\epsilon^{n}$ denote the map in the statement. We induct on $n$. For $n=0$, the theorem is trivial. Suppose that $\epsilon^{n-1}$ is a homotopy equivalence. Since the restriction $\left.\epsilon^{n}\right|_{\Sigma X} \underline{n-1}$ is homotopic to $\epsilon^{n-1}$ by Lemma 2.5, the map

$$
\left(\epsilon^{n-1}\right)^{-1} \circ \sum_{p \in P \leq n-1} \Sigma \pi_{p} \circ \rho_{p}^{n}: \Sigma X^{\underline{n}} \rightarrow \Sigma X^{\underline{n-1}}
$$

is a left homotopy inverse of the canonical map $\Sigma X^{n-1} \rightarrow \Sigma X^{n}$. Then it follows from Lemma 2.6 that the map

$$
\bar{\pi}+\sum_{p \in P \leq n-1} \Sigma \pi_{p} \circ \rho_{p}^{n}: \Sigma X^{\underline{n}} \rightarrow \Sigma\left(X^{\underline{n}} / X^{\underline{n-1}}\right) \vee \Sigma \bigvee_{p \in P \leq n-1} X(p)
$$

is a homotopy equivalence, where $\bar{\pi}: \Sigma X^{\underline{n}} \rightarrow \Sigma\left(X^{\underline{n}} / X^{\underline{n-1}}\right)$ is the projection. It is obvious that $\Sigma\left(X^{\underline{n}} / X^{\underline{n-1}}\right)=\bigvee_{p \in P^{n}} X(p)$ and the projection $\bar{\pi}$ is homotopic to $\sum_{p \in P^{n}} \Sigma \pi_{p} \circ \rho_{p}^{n}$, completing the proof.

\section{Applications of Theorem 2.3}

This section shows three applications of Theorem 2.3 which recover the results of [5] and [1].

\subsection{Product spaces}

We consider the product space $X_{1} \times \cdots \times X_{m}$. Let $[m]$ denote a finite set $\{1, \ldots, m\}$. We define a space $X$ over a lattice $2^{[m]}$, the power set of $[m]$, by

$$
X_{I}:=\prod_{i \in I} X_{i}
$$


for $I \subset[m]$. Then it is obvious that $X$ is retractile. By definition, we have $X^{\underline{n}}$ is the generalized fat wedge

$\left\{\left(x_{1}, \ldots, x_{m}\right) \in X_{1} \times \cdots \times X_{m} \mid\right.$ at least $m-n$ of the $x_{i}$ are basepoints $\}$,

and $X(I)=\bigwedge X_{i}$ for $I \subset[m]$. By Theorem 2.3 we get the standard decomposition $i \in I$

$$
\Sigma\left(X_{1} \times \cdots \times X_{m}\right) \simeq \Sigma \bigvee_{I \subset[m]} \bigwedge_{i \in I} X_{i}
$$

The case $m=2$ is the above mentioned decomposition of a product of two spaces. This decomposition of product spaces is generalized to that of polyhedral products below.

\subsection{Polyhedral products}

Let $K$ be an abstract simplicial complex on the vertex set $[m]$, and let $(\underline{X}, \underline{A}):=$ $\left\{\left(X_{i}, A_{i}\right)\right\}_{i \in[m]}$ be a collection of pairs of pointed spaces indexed by the vertex set of $K$. The polyhedral product $\mathcal{Z}_{K}(\underline{X}, \underline{A})$ is defined by

$$
\mathcal{Z}_{K}(\underline{X}, \underline{A}):=\bigcup_{\sigma \in K}(\underline{X}, \underline{A})^{\sigma} \quad\left(\subset X_{1} \times \cdots \times X_{m}\right),
$$

where $(\underline{X}, \underline{A})^{\sigma}=Y_{1} \times \cdots \times Y_{m}$, where $Y_{i}=X_{i}$ if $i \in \sigma$ and $Y_{i}=A_{i}$ otherwise. Polyhedral products are connected with several areas of mathematics as mentioned in Section 1, and this connection is actually made through homotopy invariants in many cases. So it is particularly important to describe the homotopy types of polyhedral products. In studying the homotopy types of polyhedral products, the decomposition of suspensions of polyhedral products due to Bahri, Bendersky, Cohen, and Gitler [5] is fundamental as in $[8 ; 11 ; 12]$, and we here recover this decomposition from Theorem 2.3. For $I \subset[m]$, put $K_{I}:=\{\sigma \subset I \mid \sigma \in K\}$ and $\left(\underline{X}_{I}, \underline{A}_{I}\right):=\left\{\left(X_{i}, A_{i}\right)\right\}_{i \in I}$. Then we get a polyhedral product $\mathcal{Z}_{K_{I}}\left(\underline{X}_{I}, \underline{A}_{I}\right)$ that admits an inclusion

$$
\iota_{J, I}: \mathcal{Z}_{K_{I}}\left(\underline{X}_{I}, \underline{A}_{I}\right) \rightarrow \mathcal{Z}_{K_{J}}\left(\underline{X}_{J}, \underline{A}_{J}\right)
$$

for $I \subset J \subset[m]$ by using the basepoints, where we assume $\mathcal{Z}_{K_{\varnothing}}\left(\underline{X}_{\varnothing}, \underline{A}_{\varnothing}\right)$ is a point. For $I \subset J \subset[m]$, the projection $\prod_{j \in J} X_{j} \rightarrow \prod_{i \in I} X_{i}$ induces a map

$$
\rho_{I, J}: \mathcal{Z}_{K_{J}}\left(\underline{X} J, \underline{A}_{J}\right) \rightarrow \mathcal{Z}_{K_{I}}\left(\underline{X}_{I}, \underline{A}_{I}\right)
$$

which is a retraction of the inclusion $\iota_{J, I}$. This retraction obviously satisfies the following property.

Lemma 3.1 For $I, J \subset[m]$, we have

$$
\rho_{I \cap J, I} \circ \rho_{I, I \cup J}=\rho_{I \cap J, J} \circ \rho_{J, I \cup J}
$$


The assignment

$$
I \mapsto \mathcal{Z}_{K_{I}}\left(\underline{X}_{I}, \underline{A}_{I}\right)
$$

defines a space over a lattice $2^{[m]}$ which we denote by $Z$. We define the grading of $2^{[m]}$ by the cardinality of subsets. Then the associated filtration

$$
*=Z^{\underline{0}} \subset Z^{\underline{1}} \subset \cdots \subset Z^{\underline{m}}=\mathcal{Z}_{K}(\underline{X}, \underline{A})
$$

is the fat wedge filtration which plays the fundamental role in describing the homotopy type of the special polyhedral product $\mathcal{Z}_{K}(C \underline{X}, \underline{X})$ as in [12]. We can define a space $\widehat{\mathcal{Z}}_{K}(\underline{X}, \underline{A})$ by replacing the direct product with the smash product in the definition of the polyhedral product $\mathcal{Z}_{K}(\underline{X}, \underline{A})$ above. Then for $I \subset[m]$, we have

$$
Z(I)=\widehat{\mathcal{Z}}_{K_{I}}\left(\underline{X}_{I}, \underline{A}_{I}\right) .
$$

Note that by Lemma 2.1, if each $\left(X_{i}, A_{i}\right)$ is an NDR pair, then $Z$ is $m$-cofibrant. By Lemma 3.1, $Z$ is also retractile, so by Theorem 2.3 we obtain:

Theorem 3.2 (Bahri, Bendersky, Cohen, and Gitler [5]) If $(\underline{X}, \underline{A})$ is a collection of NDR pairs, there is a homotopy equivalence

$$
\Sigma \mathcal{Z}_{K}(\underline{X}, \underline{A}) \simeq \Sigma \bigvee_{\varnothing \neq I \subset[m]} \widehat{\mathcal{Z}}_{K_{I}}\left(\underline{X}_{I}, \underline{A}_{I}\right)
$$

which is natural with respect to $(\underline{X}, \underline{A})$.

Example 3.3 Let $(\underline{X}, \underline{*})$ denote $n$-copies of a pair of a space and its basepoint $(X, *)$. Note that $\widehat{Z}_{K_{I}}(\underline{X}, \underline{*})$ is either a point or $\widehat{X}^{|I|}$ a depending on whether $I \notin K$ or $I \in K$, where $\widehat{X}^{n}$ denotes the smash product of $n$-copies of $X$. Then by Theorem 3.2 we have

$$
\Sigma \mathcal{Z}_{K}(\underline{X}, \underline{*}) \simeq \Sigma \bigvee_{\sigma \in K} \widehat{X}^{|\sigma|}
$$

which is natural with respect to $X$. This will be used below.

\subsection{Simplicial spaces}

Recall that a simplicial space $X$ is a sequence of spaces $X_{0}, X_{1}, \ldots$ equipped with face maps $d_{0}, \ldots, d_{n}: X_{n} \rightarrow X_{n-1}$ and degeneracy maps $s_{0}, \ldots, s_{n}: X_{n} \rightarrow X_{n+1}$ for all $n$ which satisfy the well-known simplicial identity. We construct a space $\underline{X}$ over a graded lattice $2^{[n]}$ for fixed $n$ from a simplicial space $X$, where the grading of the lattice $2^{[n]}$ is given by the cardinality of subsets as above. For $I \subset[n]$, we put

$$
\underline{X}_{I}:=X_{|I|}
$$


For $i \notin I$, we define $\iota_{I \cup i, I}: \underline{X}_{I} \rightarrow \underline{X}_{I \cup i}$ to be the degeneracy map $s_{j}$, where $I \cup i=$ $\left\{i_{1}<\cdots<i_{|I|+1}\right\}$ and $i_{j-1}=i$. Then we easily see that this generates a space $\underline{X}$ over $2^{[n]}$. Moreover, by the simplicial identity, $d_{j}$ is a retraction of $s_{j}$ which makes $\underline{X}$ retractile too by the simplicial identity. We next describe $\underline{X}^{\underline{m}}$ in terms of the degeneracy maps. We set

$$
S^{k}\left(X_{n}\right):=\left\{x \in X_{n} \mid x=s_{i_{1}} \cdots s_{i_{k}}(y) \text { for some } y \in X_{n-k} \text { and } i_{1}>\cdots>i_{k}\right\}
$$

for $k \geq 0$ and $S^{-1}\left(X_{n}\right)$ to be a point. By the simplicial identity $d_{i} s_{i}=1$, the map $s_{i}: X_{m} \rightarrow s_{i}\left(X_{m}\right)$ is a homeomorphism, so we have

$$
\underline{X} \frac{n-k}{\underline{n}} \cong S^{k}\left(X_{n}\right) \text {. }
$$

Then we get

$$
S^{k}\left(X_{n}\right) / S^{k+1}\left(X_{n}\right) \cong \underline{X}^{\frac{n-k}{2}} / \underline{X}^{\frac{n-k-1}{}}=\bigvee_{I \subset[n],|I|=n-k} \underline{X}(I),
$$

which was observed in [1]. Thus we obtain:

Theorem 3.4 Let $\underline{X}$ be a space over $2^{[n]}$ associated with a simplicial space $X$. If $\underline{X}$ is $n$-cofibrant, then

$$
\Sigma \underline{X}^{\underline{n}} \simeq \Sigma \bigvee_{k=0}^{n} S^{k}\left(X_{n}\right) / S^{k+1}\left(X_{n}\right) \quad \text { and } \quad S^{k}\left(X_{n}\right) / S^{k+1}\left(X_{n}\right) \cong \bigvee_{I \subset[n],|I|=n-k} \underline{X}(I),
$$

which are natural with respect to simplicial maps.

Example 3.5 Regard $[n]$ as a discrete space, and consider the standard cosimplicial structure on $\{[n]\}_{n \geq 1}$, where the indexing differs from the usual case by one. For a space $X$, we define a simplicial space $\underline{X}$ by

$$
\underline{X}_{n-1}:=\operatorname{map}([n], X) \quad\left(=X^{n}\right) .
$$

Then we have $X \underline{n-1}=X^{n}$ and $S^{k}\left(\underline{X}_{n-1}\right)$ is

$\left\{\left(x_{1}, \ldots, x_{n}\right) \in X^{n} \mid x_{i_{1}}=x_{i_{1}+1}, \ldots, x_{i_{k}}=x_{i_{k+1}}\right.$ for some $\left.1 \leq i_{1}<\cdots<i_{k} \leq n-1\right\}$,

which is the union of a special diagonal arrangement investigated below. Thus Theorem 3.4 gives a decomposition of $\Sigma\left(X^{n}\right)$ which is not the standard one in Section 3.1. This type of construction applies to the spaces of commuting elements in a Lie group as in [1]. 


\section{Diagonal arrangements}

Homotopy decompositions are fundamental powerful tools in studying the topology of subspace arrangements and their complements. Here are two examples: Ziegler and Živaljević [23] decomposed the one-point compactification of affine subspace arrangements, from which one can deduce the well known Goresky-MacPherson formula [7] on the (co)homology of the complements of affine subspace arrangements, and Bahri, Bendersky, Cohen, and Gitler [5] decompose suspensions of polyhedral products including coordinate subspace arrangements and their complements, from which one can deduce Hochster's formula on related Stanley-Reisner rings, whereas Grbic and Theriault [8] and the authors $[11 ; 12]$ studied the desuspension of the decomposition of $\Sigma \mathcal{Z}_{K}(C \underline{X}, \underline{X})$, where $(C \underline{X}, \underline{X})$ is the sequence of cones and their bases. In this section we consider a decomposition of the union of an arrangement of the following special subspaces. Fix a space $X$. For a subset $\sigma \subset[m]$, the subspace of $X^{m}$ defined by

$$
\Delta_{\sigma}(X):=\left\{\left(x_{1}, \ldots, x_{m}\right) \in X^{m} \mid x_{i_{1}}=\cdots=x_{i_{k}} \text { for }\left\{i_{1}, \ldots, i_{k}\right\}=[m]-\sigma\right\}
$$

is called the diagonal subspace of $X^{m}$ associated with $\sigma$. The arrangement of diagonal subspaces

$$
\Delta_{\sigma_{1}}(X), \ldots, \Delta_{\sigma_{k}}(X) \text { for } \sigma_{1}, \ldots, \sigma_{k} \subset[m]
$$

is called the diagonal arrangement, where it is sometimes called the hypergraph arrangement since it is determined by the hypergraph whose vertex set is $[\mathrm{m}]$ and edges are $\sigma_{1}, \ldots, \sigma_{k}$. One can regard diagonal arrangements as a generalization of the braid arrangement which corresponds to the diagonal arrangement defined by all subsets of $[m]$ with cardinality $m-2$. The topology and combinatorics of diagonal arrangements have been studied in several directions; see $[17 ; 22 ; 16 ; 14 ; 18 ; 21 ; 20]$ for example. In this paper, we are interested in the topology of the union $\Delta_{\sigma_{1}}(X) \cup \cdots \cup \Delta_{\sigma_{k}}(X)$.

We set convention and notation on diagonal arrangements. By removing the inessential part, we may assume that $\sigma_{1} \cup \cdots \cup \sigma_{k}=[\mathrm{m}]$ for the above diagonal arrangement, and it is useful to consider all diagonal subspaces included in $\Delta_{\sigma_{1}}(X), \ldots, \Delta_{\sigma_{k}}(X)$, for example, to express the union as a colimit, that is, we consider all diagonal subspaces $\Delta_{\sigma}(X)$ for $\sigma \in K$, where $K$ is a simplicial complex generated by $\sigma_{1}, \ldots, \sigma_{k}$. Then we assume that all diagonal arrangements have the form

$$
\left\{\Delta_{\sigma}(X) \mid \sigma \in K\right\}
$$

for a simplicial complex $K$ on the vertex set $[m]$. For example, the braid arrangement is the case when $K$ is the $(m-3)$-skeleton of the $(m-1)$-dimensional full simplex. 
We put

$$
\Delta_{K}(X):=\bigcup_{\sigma \in K} \Delta_{\sigma}(X)
$$

Observe that the polyhedral product $\mathcal{Z}_{K}(\underline{X}, \underline{*})$ is a subspace of $\Delta_{K}(X)$, where $(\underline{X}, \underline{*})$ denotes $m$-copies of $(X, *)$.

Labassi [18] shows that the suspension $\Sigma \Delta_{K}(X)$ decomposes into a wedge of smash products of copies of $X$ when $K$ is the $(m-d-1)$-skeleton of the $(m-1)$-simplex and $2 d>m$, in which case $\Delta_{K}(X)$ consists of all $\left(x_{1}, \ldots, x_{m}\right) \in X^{m}$ such that at least $d$-tuple of the $x_{i}$ are identical. The proof for this decomposition in [18] heavily depends on the symmetry of the skeleta of simplices, and so it cannot apply to general $K$. However, Sadok Kallel posed the following problem to the authors: is there a homotopy decomposition of $\Sigma \Delta_{K}(X)$ for $2(\operatorname{dim} K+1)<m$ which includes Labassi's decomposition? We give an affirmative answer to this question in the following:

Theorem 4.1 If $X$ has the homotopy type of a connected $C W$-complex and we have $2(\operatorname{dim} K+1)<m$, then

$$
\Sigma \Delta_{K}(X) \simeq \Sigma\left(\bigvee_{\sigma \in K} \widehat{X}^{|\sigma|} \vee \widehat{X}^{|\sigma|+1}\right),
$$

where $\widehat{X}^{k}$ is the smash product of $k$-copies of $X$ for $k>0$ and $\widehat{X}^{0}$ is a point.

As a corollary, we calculate the Euler characteristic of the complement of the diagonal arrangement $\mathcal{M}_{K}(X)=X^{m}-\Delta_{K}(X)$.

Corollary 4.2 Let $X$ be a closed connected $n$-manifold. If $2(\operatorname{dim} K+1)<m$, the Euler characteristic of $\mathcal{M}_{K}(X)$ is given by

$$
\chi\left(\mathcal{M}_{K}(X)\right)=\chi(X)^{m}-(-1)^{m n} \chi(X)\left(1+\sum_{\varnothing \neq \sigma \in K}(\chi(X)-1)^{|\sigma|}\right) .
$$

Proof Since $X$ is a compact manifold, $\Delta_{K}(X)$ is a compact, locally contractible subset of an $m n$-manifold $X^{m}$. Then by Poincaré-Alexander duality [9, Proposition 3.46], there is an isomorphism

$$
H_{i}\left(X^{m}, \mathcal{M}_{K}(X) ; \mathbb{Z} / 2\right) \cong H^{m n-i}\left(\Delta_{K}(X) ; \mathbb{Z} / 2\right),
$$

implying that $\chi\left(X^{m}, \mathcal{M}_{K}(X)\right)=(-1)^{m n} \chi\left(\Delta_{K}(X)\right)$. Since $\chi\left(\widehat{X}^{k}\right)=(\chi(X)-1)^{k}+1$ for $k \geq 1$, it follows from Theorem 4.1 that

$$
\chi\left(X^{m}, \mathcal{M}_{K}(X)\right)=(-1)^{m n} \chi(X)\left(1+\sum_{\varnothing \neq \sigma \in K}(\chi(X)-1)^{|\sigma|}\right) .
$$


Therefore the proof is completed by the equality

$$
\chi\left(X^{m}\right)=\chi\left(X^{m}, \mathcal{M}_{K}(X)\right)+\chi\left(\mathcal{M}_{K}(X)\right) .
$$

Remark 4.3 Corollary 4.2 does not hold without compactness of $X$. For example, if $X=\mathbb{R}$ (hence $n=1)$ and $K$ is the $(m-3)$-skeleton of the full $(m-1)$-simplex, $\mathcal{M}_{K}(X)$ is homotopy equivalent to $m$ ! points, implying $\chi\left(\mathcal{M}_{K}(X)\right)=m$ ! which differs from Corollary 4.2.

\section{Proof of Theorem 4.1}

The outline of the proof of Theorem 4.1 is as follows. As mentioned above, the polyhedral product $\mathcal{Z}_{K}(\underline{X}, \underline{*})$ is a subspace of $\Delta_{K}(X)$. In general the inclusion $\mathcal{Z}_{K}(\underline{X}, \underline{*}) \rightarrow \Delta_{K}(X)$ is not a fiber inclusion of a homotopy fibration, so we cannot connect properties of polyhedral products to those of $\Delta_{K}(X)$. But under the condition $2(\operatorname{dim} K+1)<m$, we can describe the inclusion to some extent, which enables us to apply the decomposition of polyhedral products from Example 3.3 to obtain Theorem 4.1.

We abbreviate $\mathcal{Z}_{K}(\underline{X}, \underline{*})$ by $X^{K}$. We start the proof of Theorem 4.1 by showing that the inclusion $X^{K} \rightarrow \Delta_{K}(X)$ is a fiber inclusion of a homotopy fibration. For this, we apply the following result of Puppe.

Lemma 5.1 (cf [6, Proposition, page 180]) Let $\left\{F_{i} \rightarrow E_{i} \rightarrow B\right\}_{i \in I}$ be an I-diagram of homotopy fibrations over a fixed connected base $B$. Then

$$
\underset{I}{\operatorname{hocolim}} F_{i} \rightarrow \underset{I}{\operatorname{hocolim}} E_{i} \rightarrow B
$$

is a homotopy fibration.

Proposition 5.2 If $X$ is connected and $2(\operatorname{dim} K+1)<m$, then there is a homotopy fibration

$$
X^{K} \rightarrow \Delta_{K}(X) \stackrel{\pi}{\rightarrow} X
$$

Proof Let $\sigma$ be a subset of $[m]$ satisfying $|\sigma|<m / 2$. For each point $\left(x_{1}, \ldots, x_{m}\right) \in$ $\Delta_{\sigma}(X)$, there is unique $x \in X$ such that more than $m / 2$ of the $x_{i}$ are equal to $x$. Then by assigning such a point $x$ to $\left(x_{1}, \ldots, x_{m}\right) \in \Delta_{\sigma}(X)$, we get a map

$$
\Delta_{\sigma}(X) \rightarrow X
$$

which is identified with the coordinate projection through a homeomorphism $\Delta_{\sigma}(X) \cong$ $X^{|\sigma|+1}$. Hence this map is a fibration with fiber $(\underline{X}, \underline{*})^{\sigma}$, and yields a diagram of 
fibrations $\left\{(\underline{X}, \underline{*})^{\sigma} \rightarrow \Delta_{\sigma}(X) \rightarrow X\right\}_{\sigma \in K}$. Thus, by Lemma 5.1, we obtain a homotopy fibration

$$
\underset{\sigma \in K}{\operatorname{hocolim}}(\underline{X}, \underline{*})^{\sigma} \rightarrow \underset{\sigma \in K}{\operatorname{hocolim}} \Delta_{\sigma}(X) \rightarrow X .
$$

For any $\tau \subset v \subset[m]$, the inclusions $(\underline{X}, \underline{*})^{\tau} \rightarrow(\underline{X}, \underline{*})^{v}$ and $\Delta_{\tau}(X) \rightarrow \Delta_{\nu}(X)$ are cofibrations, implying that there are natural homotopy equivalences

$$
\begin{aligned}
& \underset{K}{\operatorname{hocolim}}(\underline{X}, \underline{*})^{\sigma} \simeq \underset{K}{\operatorname{colim}}(\underline{X}, \underline{*})^{\sigma}=X^{K}, \\
& \underset{K}{\operatorname{hocolim}} \Delta_{\sigma}(X) \simeq \underset{K}{\operatorname{colim}} \Delta_{\sigma}(X)=\Delta_{K}(X),
\end{aligned}
$$

completing the proof.

We next show that the fibration of Proposition 5.2 splits after a suspension. To this end, we use the following.

Lemma 5.3 Consider a homotopy fibration $F \stackrel{j}{\rightarrow} E \stackrel{\pi}{\rightarrow} B$ of spaces that have the homotopy types of connected $C W$-complexes. If $\Sigma j: \Sigma F \rightarrow \Sigma E$ has a homotopy retraction, then

$$
\Sigma E \simeq \Sigma B \vee \Sigma F \vee \Sigma(B \wedge F)
$$

Remark 5.4 If we assume further that $F$ is of finite type in Lemma 5.3, it immediately follows from the Leray-Hirsch theorem that the map $\rho$ in the proof of Lemma 5.3 is an isomorphism in cohomology with any field coefficient, implying that $\rho$ is an isomorphism in the integral homology by [9, Corollary 3A.7].

Proof Let $r: \Sigma E \rightarrow \Sigma F$ be a homotopy retraction of $\Sigma j$, and let $\rho$ be the composite

$$
\Sigma E \rightarrow \Sigma E \vee \Sigma E \vee \Sigma E \stackrel{\Sigma \pi \vee r \vee \Delta}{\longrightarrow} \Sigma B \vee \Sigma F \vee \Sigma(E \wedge E) \stackrel{1 \vee 1 \vee(\pi \wedge r)}{\longrightarrow} \Sigma B^{\vee},
$$

where $A^{\vee}=A \vee F \vee(A \wedge F)$ for a space $A$. Since $\Sigma E$ and $\Sigma B \vee \Sigma F \vee \Sigma(B \wedge F)$ have the homotopy types of simply connected CW-complexes, it is sufficient to show that $\rho$ is an isomorphism in homology by the $\mathrm{JHC}$ Whitehead theorem. We first consider the special case when there is a fiberwise homotopy equivalence $\theta: B \times F \rightarrow E$ over $B$. Then it is straightforward to see that

$$
\rho_{*} \circ \theta_{*}(b \times f)=b \times \hat{\theta}_{*}(f)+\sum_{\left|b_{i}\right|<|b|} b_{i} \times f_{i}
$$

for singular chains $b, b_{i}$ in $B$ and $f, f_{i}$ in $F$, where we omit writing the suspension isomorphism of homology and $\hat{\theta}$ is a self-homotopy equivalence of $F$ given by the composite

$$
\Sigma F \stackrel{j}{\rightarrow} \Sigma(B \times F) \stackrel{\theta}{\rightarrow} \Sigma E \stackrel{r}{\rightarrow} \Sigma F
$$


This readily implies that the map $\rho \circ \theta$ is an isomorphism in homology, and then so is $\rho$. For nonconnected $B$, the above is also true if we assume that $r$ is a homotopy retraction of the suspension of the fiber inclusion on each component of $B$. We next consider the general case. Let $B_{n}$ be the $n$-skeleton of $B$, and let $E_{n}=\pi^{-1}\left(B_{n}\right)$. We prove that the restriction $\left.\rho\right|_{\Sigma E_{n}}: \Sigma E_{n} \rightarrow \Sigma B_{n}^{\vee}$ is an isomorphism in homology by induction on $n$. Since $B$ is connected, $j$ is homotopic to the composite

$$
F \simeq \pi^{-1}(b) \stackrel{\text { incl }}{\longrightarrow} E
$$

for any $b \in B$. Then $\left.\rho\right|_{\Sigma E_{0}}: \Sigma E_{0} \rightarrow \Sigma B_{0}^{\vee}$ is an isomorphism in homology. Consider the following commutative diagram of homology exact sequences:

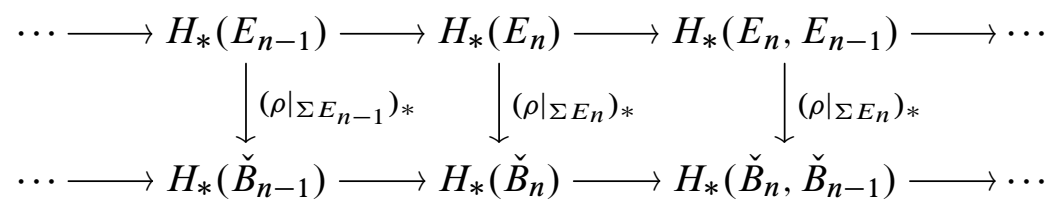

By the induction hypothesis, $\left(\left.\rho\right|_{\Sigma E_{n-1}}\right)_{*}$ is an isomorphism. Since $B_{n-1}$ is a subcomplex of $B_{n}$, there is a neighborhood $U \subset B_{n}$ of $B_{n-1}$ which deforms onto $B_{n-1}$. Then there is a commutative diagram

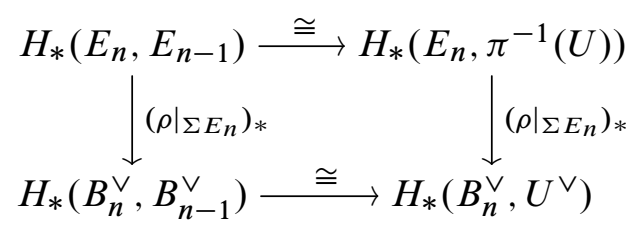

where the basepoint is taken in $B_{n-1}$. On the other hand, by the excision isomorphism, there is a commutative diagram

$$
\begin{aligned}
& H_{*}\left(E_{n}, \pi^{-1}(U)\right) \stackrel{\cong}{\cong} H_{*}\left(E_{n}-E_{n-1}, \pi^{-1}(U)-E_{n-1}\right) \\
& \left\lfloor( \rho | _ { \Sigma E _ { n } } ) _ { * } \left\lfloor\left(\left.\rho\right|_{\Sigma\left(E_{n}-E_{n-1}\right)}\right)_{*}\right.\right. \\
& H_{*}\left(B_{n}^{\vee}, U^{\vee}\right) \longleftarrow \cong H_{*}\left(\left(B_{n}-B_{n-1}\right)^{\vee},\left(U-B_{n-1}\right)^{\vee}\right)
\end{aligned}
$$

where the basepoint is taken in $U-B_{n-1}$ and is connected by a path to the former basepoint in $B_{n-1}$. Since each connected component of $B_{n}-B_{n-1}$ is contractible, $E_{n}-E_{n-1}$ is fiberwise homotopy equivalent to $\left(B_{n}-B_{n-1}\right) \times F$ over $B_{n}-B_{n-1}$, and then so is also $\pi^{-1}(U)-E_{n-1}$ to $\left(U-B_{n-1}\right) \times F$ over $U-B_{n-1}$. As in the 0 -skeleton case, we see that $r$ restricts to a homotopy retraction of the suspension of the fiber inclusion on each component of $B_{n}-B_{n-1}$. Then by the above trivial fibration case, we obtain that the map $\left(\left.\rho\right|_{\Sigma\left(E_{n}-E_{n-1}\right)}\right)_{*}$ in (5-3) is an isomorphism. 
Although the basepoints used in (5-2) and (5-3) are distinct, they are connected by a path. In particular, we can juxtapose (5-2) and (5-3) to obtain that the right $\left(\left.\rho\right|_{\Sigma E_{n}}\right)_{*}$ in (5-1) is an isomorphism. Thus by the five-lemma, the middle $\left(\left.\rho\right|_{\Sigma E_{n}}\right)_{*}$ in (5-1) is an isomorphism. We finally take the colimit to get that the map $\rho$ is an isomorphism in homology as desired, completing the proof.

To apply Lemma 5.3 to the fibration of Proposition 5.2, we construct a homotopy retraction of a suspension of the fiber inclusion $j: X^{K} \rightarrow \Delta_{K}(X)$. We first consider a special case.

Proposition 5.5 If $X$ is an $H$-space that has the homotopy type of a $C W$-complex and $2(\operatorname{dim} K+1)<m$, then the fibration of Proposition 5.2 is trivial.

Proof Consider the map

$$
\varphi: X \times X^{K} \rightarrow \Delta_{K}(X), \quad\left(x,\left(x_{1}, \ldots, x_{m}\right)\right) \mapsto\left(x x_{1}, \ldots, x x_{m}\right) .
$$

Then $\varphi$ satisfies a homotopy commutative diagram

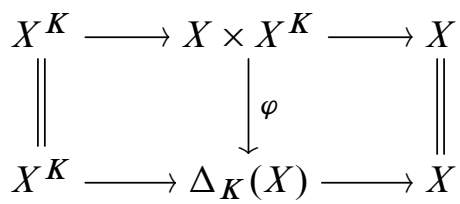

in which two rows are homotopy fibrations. Then it follows from the homotopy exact sequence that $\varphi$ is a weak homotopy equivalence, hence a homotopy equivalence by the J HC Whitehead theorem.

We set notation. Put $\widehat{X}^{K}=\bigvee_{\sigma \in K} \widehat{X}^{|\sigma|}$, and let $\epsilon: \Sigma X^{K} \stackrel{\simeq}{\longrightarrow} \Sigma \widehat{X}^{K}$ denote the homotopy equivalence of Example 3.3. Then a map $f: X \rightarrow Y$ induces maps $f^{K}: X^{K} \rightarrow Y^{K}$ and $\hat{f}^{K}: \widehat{X}^{K} \rightarrow \widehat{Y}^{K}$ which satisfy a commutative diagram

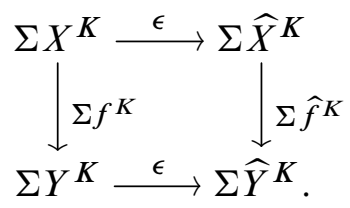

Proposition 5.6 If $X$ has the homotopy type of a connected CW-complex and $2(\operatorname{dim} K+1)<m$, then the inclusion $j: X^{K} \rightarrow \Delta_{K}(X)$ has a homotopy retraction after a suspension. 
Proof Let $E: X \rightarrow \Omega \Sigma X$ be the suspension map. Since $\Sigma E$ has a retraction, we easily see that the induced map $\Sigma \widehat{E}^{K}: \Sigma \widehat{X}^{K} \rightarrow \Sigma \widehat{\Omega \Sigma X}^{K}$ has a retraction, say $r$. Then we get a homotopy commutative diagram

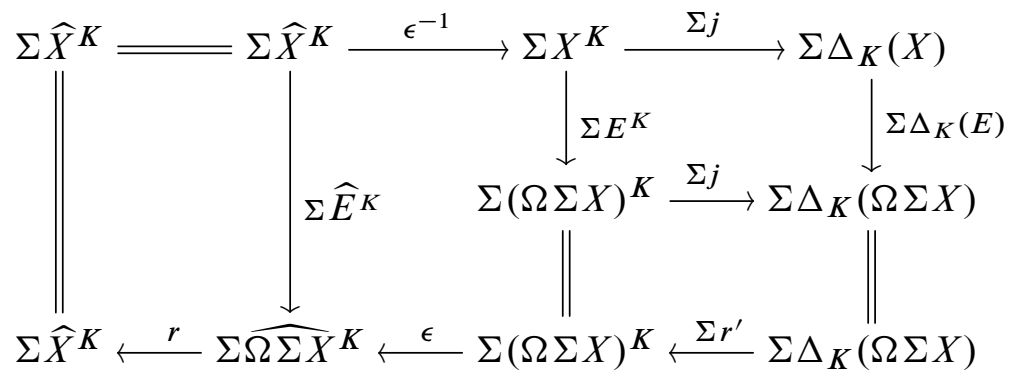

where $\Delta_{K}(E): \Delta_{K}(X) \rightarrow \Delta_{K}(\Omega \Sigma X)$ is induced from $E$ and $r^{\prime}$ is obtained by Proposition 5.5. Thus the composite

$\Sigma \Delta_{K}(X) \stackrel{\Sigma \Delta_{K}(E)}{\longrightarrow} \Sigma \Delta_{K}(\Omega \Sigma X) \stackrel{\Sigma r^{\prime}}{\longrightarrow} \Sigma(\Omega \Sigma X) \stackrel{{ }^{\prime}}{\rightarrow} \Sigma \widehat{\Omega \Sigma X}{ }^{K} \stackrel{r}{\rightarrow} \Sigma \widehat{X}^{K} \stackrel{\epsilon^{-1}}{\longrightarrow} \Sigma X^{K}$ is the desired homotopy retraction.

Proof of Theorem 4.1 Combine Example 3.3, Proposition 5.2, Lemma 5.3 and Proposition 5.6.

\section{References}

[1] A Adem, A Bahri, M Bendersky, F R Cohen, S Gitler, On decomposing suspensions of simplicial spaces, Bol. Soc. Mat. Mexicana 15 (2009) 91-102 MR2757688

[2] A Adem, F R Cohen, Commuting elements and spaces of homomorphisms, Math. Ann. 338 (2007) 587-626 MR2317932

[3] A Adem, F R Cohen, J M Gómez, Stable splittings, spaces of representations and almost commuting elements in Lie groups, Math. Proc. Cambridge Philos. Soc. 149 (2010) 455-490 MR2726729

[4] A Adem, F R Cohen, E Torres Giese, Commuting elements, simplicial spaces and filtrations of classifying spaces, Math. Proc. Cambridge Philos. Soc. 152 (2012) 91-114 MR2860418

[5] A Bahri, M Bendersky, F R Cohen, S Gitler, The polyhedral product functor: a method of decomposition for moment-angle complexes, arrangements and related spaces, Adv. Math. 225 (2010) 1634-1668 MR2673742

[6] E D Farjoun, Cellular spaces, null spaces and homotopy localization, Lecture Notes in Mathematics 1622, Springer, Berlin (1996) MR1392221 
[7] M Goresky, R MacPherson, Stratified Morse theory, Ergeb. Math. Grenzgeb. 14, Springer, Berlin (1988) MR932724

[8] J Grbic, S Theriault, The homotopy type of the complement of a coordinate subspace arrangement, Topology 46 (2007) 357-396 MR2321037

[9] A Hatcher, Algebraic topology, Cambridge University Press, Cambridge (2002) MR1867354

[10] P Hilton, G Mislin, J Roitberg, On co- H-spaces, Comment. Math. Helv. 53 (1978) 1-14 MR483528

[11] K Iriye, D Kishimoto, Decompositions of polyhedral products for shifted complexes, Adv. Math. 245 (2013) 716-736 MR3084442

[12] K Iriye, D Kishimoto, Fat wedge filtrations and decomposition of polyhedral products, preprint (2015) arXiv:1412.4866

[13] I M James, On H-spaces and their homotopy groups, Quart. J. Math. Oxford Ser. 11 (1960) 161-179 MR0133132

[14] S Kallel, I Saihi, Homotopy groups of diagonal complements, preprint (2013) arXiv: 1306.6272

[15] Y Kamiyama, S Tsukuda, The configuration space of the $n$-arms machine in the Euclidean space, Topology Appl. 154 (2007) 1447-1464 MR2310477

[16] S Kim, Shellable complexes and topology of diagonal arrangements, Discrete Comput. Geom. 40 (2008) 190-213 MR2438924

[17] D N Kozlov, A class of hypergraph arrangements with shellable intersection lattice, J. Combin. Theory Ser. A 86 (1999) 169-176 MR1682970

[18] F Labassi, Sur les diagonales épaisses et leurs complémentaires, J. Homotopy Relat. Struct. 10 (2015) 375-400 MR3385691

[19] J Lillig, A union theorem for cofibrations, Arch. Math. (Basel) 24 (1973) 410-415 MR0334193

[20] MS Miller, Massey products and k-equal manifolds, Int. Math. Res. Not. 2012 (2012) 1805-1821 MR2920831

[21] M Miller, M Wakefield, Formality of Pascal arrangements, Proc. Amer. Math. Soc. 139 (2011) 4461-4466 MR2823091

[22] I Peeva, V Reiner, V Welker, Cohomology of real diagonal subspace arrangements via resolutions, Compositio Math. 117 (1999) 99-115 MR1693007

[23] G M Ziegler, R T Živaljević, Homotopy types of subspace arrangements via diagrams of spaces, Math. Ann. 295 (1993) 527-548 MR1204836 
Department of Mathematics and Information Sciences, Osaka Prefecture University Sakai 599-8531, Japan

Department of Mathematics, Kyoto University

Kyoto 606-8502, Japan

kiriye@mi.s.osakafu-u.ac.jp, kishi@math.kyoto-u.ac.jp

Received: 15 December $2014 \quad$ Revised: 29 June 2015 
